\title{
Influence of the convective flux perturbation on stellar oscillations: application to $\delta$ Scuti and $\gamma$ Doradus stars
}

\author{
A. Grigahcène ${ }^{1}$, M-A. Dupret ${ }^{1}$, R. Garrido ${ }^{1}, M$. Gabriel $^{2}$ and R. Scuflaire ${ }^{2}$ \\ ${ }^{1}$ Instituto de Astrofísica de Andalucía-CSIC, Apartado 3004, 18080 Granada, Spain \\ ${ }^{2}$ Institut d'Astrophysique et de Géophysique de l'Université de Liège, Belgium
}

\begin{abstract}
We present a theory of convection-oscillation interaction. In our nonradial nonadiabatic pulsation code, the variation of the convective flux (radial and transversal components) is taken into account, following the theory of M. Gabriel, within the mixing length approach. We explore the influence of the convective flux variation on mode stability near the red-edge of the $\delta$ Scuti instability strip and the excitation mechanisms of $\gamma$ Doradus stars.

\section{Introduction}

The determination of theoretical instability strips is of great interest in the study of pulsating stars, because their confrontation with observations enables us to test our knowledge of stellar interiors and our understanding of the driving mechanisms of these stars. Using the timedependent convection theory of Gabriel $(1974,1996,1998,2000)$, we study the influence of the convective flux perturbation on stellar oscillations. We present instability strips for $\delta$ Scuti and $\gamma$ Doradus stars.
\end{abstract}

\section{Results}

We have implemented the perturbation of the convective flux in the linear non-adiabatic code MAD (Dupret et al. 2002). In order to determine the theoretical instability strips, a large number of evolutionary tracks were computed, with masses ranging from 1.4 to $2.2 M_{\odot}$, and with different values of the MLT parameter $\alpha$. Then, we studied the stability of the modes in the appropriate frequency range.

\section{$\delta$ Scuti instability strip}

Contrary to the calculations with frozen convection, with our time-dependent convection treatment we are able to reproduce the red edge of the $\delta$ Scuti instability strip, for radial as well as for non-radial modes.

In the left panel of Fig. 1, we present the theoretical instability strip obtained for radial modes, for models with the solar calibrated value $\alpha=1.8$. Each curve corresponds to the blue or red edge of a mode of given radial order $n$. Labels permit to identify the modes (e.g. $\mathrm{p}_{4 \mathrm{R}}$ for the red edge of the $\mathrm{p}_{4}$ mode). As the radial order of the modes increases, the blue and red edges are displaced towards higher $T_{\text {eff }}$. The points correspond to the position of observed $\delta$ Scuti stars, as taken from the catalogue of Rodriguez et al. (2000), using the calibrations of Moon \& Dworetsky (1985). 

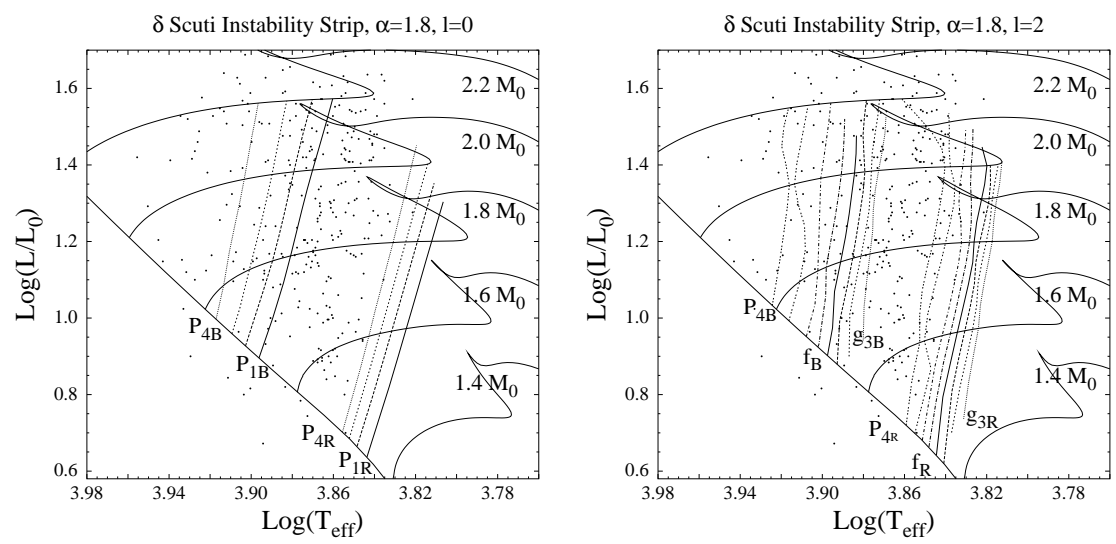

Figure 1: Left panel: Blue and red edges of the $\delta$ Scuti theoretical instability strip for models with $\alpha=1.8$ and radial modes from $p_{1}$ to $p_{4}$. Right panel: Blue and red edges of the $\delta$ Scuti theoretical instability strip for models with $\alpha=1.8$ and $\ell=2$ modes from $g_{3}$ to $p_{4}$. The points correspond to observations.

In the right panel of Fig. 1, we present the theoretical instability strip obtained for $\ell=2$ modes, for models with $\alpha=1.8$.

\section{$\gamma$ Doradus instability strip}

The driving of the high-order gravity modes of $\gamma$ Doradus stars can also be explained by our time-dependent convection models. In the left panel of Fig. 2, we show the periods of all the unstable $\ell=1$ gravity modes obtained for models of $1.6 M_{\odot}$ with $\alpha=2$, as a function of the effective temperature. Each cross corresponds to an unstable mode. As can be seen, the periods of those modes correspond to the typical observed periods of $\gamma$ Doradus stars. Moreover, we see in the bottom of this figure that our models have also unstable p-modes, typical of $\delta$ Scuti stars. In the right panel of Fig. 2, we show the theoretical instability strips of $\gamma$ Doradus $\ell=1$ modes obtained for three families of models with different values of the MLT parameter $\alpha: 1,1.5$ and 2. In this case, we give global instability strips and not individual ones for each mode. For any model inside the instability strip, at least one unstable high order g-mode is found; outside it all the g-modes are found to be stable. The small circles correspond to the observed positions of bona fide $\gamma$ Doradus stars from the catalogue of Handler (2002); their effective temperatures are taken from Kaye et al. (1999) who used the calibrations of Villa (1998). As for $\delta$ Scuti stars, we see that the theoretical predictions are very sensitive to $\alpha$. In agreement with Guzik et al. (2000), we find that the depth of the convective envelope plays the major role in the driving of $\gamma$ Doradus g-modes. This explains the high sensitivity of our results to $\alpha$.

\section{Conclusion}

Including the perturbation of the convective flux, following Gabriel's theory, we obtained theoretical instability strip of $\delta$ Scuti and $\gamma$ Doradus stars. For $\delta$ Scuti stars, we succeed to reproduce both the blue and red edges, for radial as well as for non-radial modes. The location of the theoretical red edge appears to be very sensitive to the value of the MLT parameter $\alpha$. With the solar calibrated value $\alpha=1.8$, a good agreement with observations is found. We 

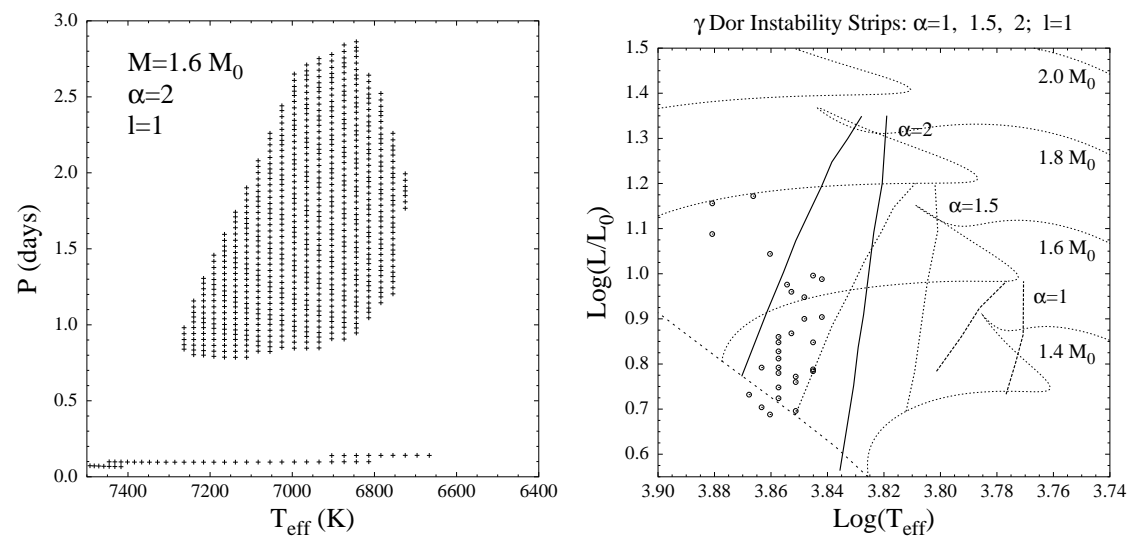

Figure 2: Left panel: Periods (in days) of all the unstable $\ell=1$ gravity modes obtained for models of 1.6 $M_{\odot}$ with $\alpha=2$, as a function of the effective temperature. Each cross corresponds to a given g-mode. Right panel: $\gamma$ Doradus theoretical instability strips for $\ell=1$ modes, for three families of models with different values of $\alpha: 1,1.5$ and 2. The small circles correspond to observations of bona fide $\gamma$ Doradus stars.

obtained also theoretical instability strips for the $\gamma$ Doradus g-modes. As for $\delta$ Scuti stars, the theoretical instability strips of $\gamma$ Doradus stars are very sensitive to the value of the MLT parameter $\alpha$. We obtain good agreement with observations for models with $\alpha=2$.

Finally, for some models, we found a mixture of unstable $\mathrm{p}$ - and g-modes, therefore predicting objects able to show simultaneous $\gamma$ Doradus and $\delta$ Scuti pulsational behaviour.

Acknowledgments. MAD acknowledges support through a European Community Marie Curie Fellowship. AG and RG acknowledge financial support from the program ESP20014528-PE.

\section{References}

Dupret, M.-A., De Ridder, J., Neuforge, C., et al. 2002, A\&A, 385, 563

Gabriel, M., Scuflaire, R., Noels, A. 1974, Bul. Ac. Roy. Belgique, Classe des Sciences 60, 866

Gabriel, M. 1996, Bull. Astron. Soc. of India, 24, 233

Gabriel, M. 1998, In: SOHO 6/GONG 98 Workshop, Boston, Massachusetts, p. 863

Gabriel, M. 2000, A\&A, 353, 399

Guzik, J. A., Kaye, A. B., Bradley, P. A., et al. 2000, ApJ, 542, L57

Handler, G. 2002, http://www.astro.univie.ac.at/ dsn/gerald/gdorlist.html

Kaye, A. B., Handler, G., Krisciunas, K., et al. 1999, PASP, 111, 840

Moon, T. T., \& Dworetsky, M. M. 1985, MNRAS, 217, 305

Rodriguez, E., Lopez-Gonzalez, M. J., Lopez de Coca, P. 2000, A\&As, 144, 469

Villa, P. 1998, M.Sc. thesis, Univ. Vienna 